\title{
Experiencia docente en la enseñanza de investigación en estudiantes de Medicina de la materia Pediatría
}

\author{
Training students on research, while studying Pediatrics in \\ the School of Medicine. Teaching experience
}

\section{Dr. Alfredo Eymann ${ }^{a}$, Dra. Lucía Pereza ${ }^{a}$ Dr. Julio Busanichea , Dr. Nicolás Cacchiarelli ${ }^{a}$, Dra. Clara Ceriani Cernadas ${ }^{a}$ y Dr. Carlos Wahren ${ }^{a}$}

\section{RESUMEN}

La investigación es importante durante la formación universitaria. El objetivo de este trabajo fue describir la enseñanza de un ejercicio de investigación clínica y su percepción por parte de los estudiantes.

Estudiantes de Medicina realizaron un trabajo grupal coordinados por un tutor. Se evaluó la importancia que les daban los alumnos al aprendizaje de la investigación y la satisfacción de haber participado en una escala de 1 a 10.

Ochenta y un estudiantes desarrollaron 14 ejercicios de investigación. El 44,4\% refirió haber recibido formación en metodología de la investigación y el 11,1\% había participado de algún estudio de investigación. Se le asignó un puntaje de 8 a la importancia de investigar y 9 a la satisfacción de haber participado de la actividad. Los aspectos del ejercicio de investigación clínica percibidos como positivos fueron que favoreció el trabajo en equipo y que ayudó a comprender cómo se realizaba un estudio de investigación. Palabras clave: investigación, estudiantes de Medicina, pediatría, enseñanza.

\section{ABSTRACT}

Research is important during university education. The objective was to describe the teaching of an exercise in clinical research and its perception by students.

Medical students conducted a research work in groups coordinated by a tutor. We evaluated the importance the students gave to research learning and weassessed the satisfaction of having participated in the activity in a scale from 1 to 10 . Eighty-one students developed 14 research exercises, $44.4 \%$ reported having received training in research methodology before and $11.1 \%$ had participated in some research study. They assigned a score of 8 out of 10 to the importance of research and 9 out of 10 to the satisfaction of having participated in the activity. The aspects of the exercise in clinical research perceived as positive were that it favoured teamwork and helped students to understand how to conduct a research study.

Key words: research, student, medical, pediatrics, teaching.

Financiamiento:

Ninguno.

Conflicto de intereses:

Ninguno que declarar.

Recibido: 17-6-2016

Aceptado: 16-11-2016
Cómo citar: Eymann A, Pérez L, Busaniche J, et al Experiencia docente en la enseñanza de investigación en estudiantes de Medicina de la materia Pediatría. Arch Argent Pediatr 2017;115(2):200-204.

\section{INTRODUCCIÓN}

La investigación es la actividad orientada a la obtención de nuevos conocimientos y su aplicación para la solución de problemas o preguntas de carácter científico. La investigación clínica consiste en hacerse preguntas sobre la práctica cotidiana, definir problemas y elegir un método científico para responderlas. ${ }^{1}$

Una de las funciones de las Universidades modernas es la construcción de nuevo conocimiento: diversos grupos académicos participan en espacios de intercambio e investigación para su generación. ${ }^{2}$ A partir de la Reforma Educativa humboldtiana del siglo XIX, se asigna a la educación universitaria una tarea clave para producir ciencia y conocimiento mediante la investigación científica. ${ }^{3}$

Los estudiantes universitarios se benefician de la investigación al adquirir conocimientos más profundos si están involucrados en ella y también mediante el aprendizaje basado en la investigación. ${ }^{4}$ Además, comprender el proceso de la investigación favorece la lectura crítica de los estudios de investigación para su práctica futura. ${ }^{5}$

Se han realizado diversos estudios en Latinoamérica que evalúan la formación universitaria en investigación y la participación de los estudiantes en proyectos científicos. ${ }^{5-13}$

Un estudio peruano realizado mediante encuestas a estudiantes de Medicina informó que consideraban la actividad científica como muy importante, y el principal 
determinante era la difusión del conocimiento generado. Dentro de las limitaciones referidas por los estudiantes, se mencionaban la falta de valoración personal del trabajo realizado, la ausencia de cultura de publicación y la falta de capacitación.?

Otro estudio peruano realizado mediante encuestas a estudiantes de Medicina describió que el $66,3 \%$ refirieron no haber recibido curso extracurricular de investigación y el 59,6\% no había realizado ningún trabajo de investigación. ${ }^{8}$

En Alemania, los estudiantes de Medicina tienen que presentar una tesis para recibir el título. En un estudio realizado para evaluar el aporte de la investigación de grado a la producción científica en este país, se encontró que, en el $28 \%$ de los artículos publicados indexados por dicha facultad, aparecían estudiantes como autores, y, en el $7,8 \%$, los estudiantes eran primeros autores. El 66\% de las investigaciones llevadas a cabo por estudiantes de Medicina eran publicadas en revistas indexadas. ${ }^{9}$

El currículo de la carrera de Medicina de la Universidad de Buenos Aires incluye la materia Metodología de la Investigación para cursar solo en forma electiva. La actividad de investigación durante la carrera de grado en la carrera de Medicina es baja tanto en Argentina como en Latinoamérica. ${ }^{10}$

En vista de la importancia de la investigación en la formación universitaria y de que no se han encontrado estudios en nuestro medio que muestren la participación de estudiantes en el proceso de investigación durante la carrera de grado, nos proponemos describir una experiencia docente en la enseñanza de un ejercicio de investigación clínica y la percepción de los estudiantes durante la materia Pediatría de la carrera de Medicina de la Universidad de Buenos Aires.

\section{POBLACIÓN Y MÉTODOS}

La materia Pediatría de la carrera de Medicina de la Universidad de Buenos Aires se enseña en un ámbito hospitalario durante 10 semanas. Se desarrolla de lunes a viernes entre las 8.30 y las 13 con una carga semanal de 22,5 h. Además de las actividades teóricas y prácticas curriculares, nuestra unidad académica en el Hospital Italiano de Buenos Aires, desarrolla un ejercicio de investigación clínica grupal durante toda la cursada.

Al inicio de la materia, se dictó una clase de $2 \mathrm{~h}$ para explicar los objetivos del ejercicio de investigación clínica y los fundamentos de metodología de la investigación.
Los estudiantes se reunieron en 3 grupos de 5 a 7 integrantes coordinados por un tutor. Este fue un docente con experiencia en investigación, que coordinó encuentros con los grupos a través de reuniones presenciales o por correo electrónico para el desarrollo del ejercicio de investigación.

Se definió como ejercicio de investigación clínica a lo siguiente: a) identificar una pregunta; b) realizar una introducción que fundamentara bibliográficamente por qué era importante y el estado del conocimiento del tema; c) describir en "material y métodos" diseño, población, muestra, criterios de inclusión y/o exclusión, encuesta y métodos estadísticos; d) trabajo de campo con pacientes en la toma de la encuesta; e) diseño de base de datos y análisis de los resultados; f) presentación del proyecto de investigación como un documento en formato papel y en formato de presentación oral frente al equipo docente y los compañeros en 7 minutos.

Cada grupo dispuso de $2 \mathrm{~h}$ por día durante 3 semanas no consecutivas en el horario de actividades prácticas para realizar el ejercicio. La participación en el desarrollo del trabajo de investigación formó parte de la evaluación de la materia.

En el ejercicio de investigación clínica, se evaluaron las siguientes variables: título, número de autores, número de citas que fundamentaban la introducción, objetivo (sí-no), diseño del estudio (sí-no), descripción de metodología (síno), criterios de inclusión y/o exclusión (sí-no), tamaño muestral, uso de estadística (no usó, descriptiva o analítica), número de páginas.

Se evaluaron en forma anónima las siguientes variables: sexo, edad y número de materias aprobadas, haber recibido metodología de la investigación o haber participado en un proyecto de investigación, puntaje asignado a la importancia de aprender investigación durante la carrera y satisfacción de la experiencia realizada (1: mínima; 10: máxima) y aspectos positivos y negativos referidos en texto libre (ver el Anexo en formato electrónico).

Las variables categóricas se describieron en porcentajes y las variables continuas, en mediana con intervalo intercuartílico (se evaluó la normalidad con la prueba de Shapiro-Wilk y gráficos). Las variables cualitativas se agruparon en categorías.

\section{RESULTADOS}

Participaron 81 estudiantes en 14 ejercicios de investigación clínica. Todos tomaron parte en las 
diferentes etapas del proceso del ejercicio (definir el objetivo, diseño, trabajo de campo, diseño de base de datos y análisis de los resultados).

Todos presentaron título, introducción con fundamento bibliográfico, objetivos, diseño de estudio, uso de encuesta, trabajo de campo y análisis estadístico (Tablas 1 y 2).

Los estudiantes fueron mujeres en el 77,8\% y todos cursaban el último año de la carrera. El 44,4\% reconoció haber recibido formación en metodología de la investigación y solo $11,1 \%$ había participado de algún estudio de investigación. Se le asignó una mediana de puntaje de 8 a la importancia de aprender a investigar durante la carrera de Medicina y de 9 a la satisfacción de haber participado de la actividad del ejercicio de investigación clínica (Tabla 3).

TaBla 1. Características de los ejercicios de investigación clínica $(n=14)$

\begin{tabular}{|c|c|c|}
\hline \multicolumn{2}{|l|}{ Número de estudiantes por equipo* } & $5(5-6)$ \\
\hline \multicolumn{2}{|l|}{ Número de páginas* } & $10(8-12)$ \\
\hline \multicolumn{2}{|l|}{ Título (\%) } & 100 \\
\hline \multicolumn{2}{|l|}{ Definición de objetivo (\%) } & 100 \\
\hline \multicolumn{2}{|l|}{ Descripción de diseño: corte transversal (\%) } & 100 \\
\hline \multirow[t]{2}{*}{ Criterios de inclusión y/o exclusión (\%) } & SÍ & 71,6 \\
\hline & $\mathrm{NO}$ & 21,4 \\
\hline \multicolumn{2}{|l|}{ Uso de encuesta (\%) } & 100 \\
\hline \multicolumn{2}{|l|}{ Número de preguntas de la encuesta* } & $15(12-19)$ \\
\hline \multicolumn{2}{|l|}{ Número de pacientes* } & $31,5(30-50)$ \\
\hline \multirow[t]{2}{*}{ Uso de estadística (\%) } & Descriptiva & a 78,6 \\
\hline & Analítica & 21,4 \\
\hline \multicolumn{2}{|l|}{ Número de citas bibliográficas* } & $8(6-10)$ \\
\hline
\end{tabular}

* Mediana e intervalo intercuartílico: 25-75.
Los aspectos percibidos como positivos del ejercicio de investigación clínica se agruparon en las siguientes categorías: a) 50\% refirió que favorecía el trabajo en equipo; b) $50 \%$, que ayudaba a comprender cómo se realizaba un estudio de investigación; c) $28 \%$ destacó la posibilidad de realizar el trabajo de campo en contacto con pacientes; d) 17\% refirió el aprender a consultar bibliografía; y e) 17\% mencionó el aprender a construir bases de datos y utilizar pruebas estadísticas. Además, refirieron la importancia de realizar una presentación oral de un estudio de investigación, realizar gráficos $\mathrm{y}$ tablas, aprender a escribir un proyecto e interpretar estudios de investigación.

Los aspectos percibidos como negativos se agruparon en las siguientes categorías: a) 67\% mencionó que restaba tiempo para el estudio de la materia; b) 17\% destacó dificultades en los acuerdos del trabajo grupal; y c) 11\% refirió que faltaron clases con contenido teórico en investigación.

TABla 3. Características de los estudiantes y sus percepciones en relación con el ejercicio de investigación clínica $(n=81)$

\begin{tabular}{lc}
\hline Edad $^{*}$ & $25(24-27)$ \\
Sexo femenino (\%) & 77,8 \\
Número de materias aprobadas* & $31,5(30-34)$ \\
Formación en metodología de la investigación (\%) & 44,4 \\
Participación en estudios de investigación (\%) & 11,1 \\
Importancia de aprender investigación* & $8(8-8)$ \\
Satisfacción por la actividad de investigación* & $9(8-10)$ \\
\hline
\end{tabular}

* Mediana e intervalo intercuartílico: 25-75.

TABLA 2. Títulos de los ejercicios de investigación clínica

1 Creencias y conocimientos de los adolescentes acerca de los métodos preventivos en las relaciones sexuales

2 ¿Cuál es la adherencia a la lactancia materna en madres que consultan en pediatría ambulatoria en un hospital de comunidad de alta complejidad?

3 Tasa de cumplimiento de vacunación DPTa y HPV en niños

4 Evaluación del cumplimiento de la lactancia materna exclusiva

5 Intoxicaciones más frecuentes en los niños de 0 a 16 años de edad

6 Adolescentes y anticoncepción de emergencia

7 Consumo de tabaco y alcohol en adolescentes de 15 a 17 años de escuelas públicas de la Ciudad Autónoma de Buenos Aires

8 Lesiones prevenibles en la infancia

9 Lactancia y factores de introducción de otras leches

10 Automedicación en población pediátrica

11 Aplicación de vacunas extra calendario oficial en población infantil menor de 36 meses

12 Estrategias de crianza, ¿es todavía el chirlo un método elegido por los padres?

13 Conductas tomadas por los padres de los niños frente a un cuadro de diarrea aguda

14 Prevalencia de colecho y sueño seguro 


\section{DISCUSIÓN}

La experiencia docente en la enseñanza de investigación con estudiantes de la materia Pediatría fue percibida en forma satisfactoria. Como ocurre en otras partes del mundo, hay mayor proporción de estudiantes de Medicina mujeres. ${ }^{14-22}$

El número de estudiantes que refirió haber tenido formación en metodología de la investigación durante la carrera fue menor que el reportado en la literatura latinoamericana.

Díaz Vélez y col., ${ }^{11}$ realizaron un estudio mediante encuestas a estudiantes de Medicina $(n=1484)$ en el que se reportó que el 77\% había participado alguna vez en algún proyecto de investigación y el 34\% había asistido a algún curso extracurricular de metodología de la investigación. En ese estudio, los aspectos asociados con mayor dificultad estaban relacionados con el diseño y el análisis estadístico.

En nuestro estudio, la mayor dificultad reportada fue que les quitó tiempo para el estudio de la materia. La poca dificultad referida al diseño y/o análisis podría deberse a la tarea de acompañamiento de los tutores. ${ }^{23}$

Un estudio realizado por Ángel-Isaza y col., ${ }^{12}$ informó que el 98\% de los estudiantes creía que era importante investigar, pero el $79 \%$ no realizaba actividades de investigación y refería la falta de tiempo como la principal dificultad, seguida de la falta de asesoría.

Los estudiantes percibieron como aspecto positivo en el desarrollo de la actividad que favoreció el trabajo en equipo, lo cual era importante tanto para las actividades de investigación como para la práctica clínica. Además, al reconocer dificultades en los acuerdos del trabajo grupal y aprender a reconocer esta situación y su resolución coordinados por un tutor, podía ser útil en futuros desacuerdos en trabajos de equipo.

Los ejercicios de investigación realizados por los estudiantes cumplieron con el contenido recomendado (introducción, objetivos, materiales y métodos, y redacción de resultados) ${ }^{24-25}$ Los temas elegidos para investigar eran temas de atención primaria en niños y adolescentes, formaban parte del programa currículo o programa de la materia ${ }^{26}$ y se ajustaban al perfil del médico egresado de la Universidad de Buenos Aires. ${ }^{27}$

A diferencia de la modalidad de fin de carrera en Argentina, en Perú, el 73\% de los estudiantes finaliza la carrera con la realización de una tesis. ${ }^{11}$
Jorge Fernández y col., ${ }^{13}$ realizaron un estudio para determinar las características de la formación en investigación durante la carrera de Medicina y encontraron que era deficiente para el desarrollo de habilidades en la tarea investigativa, a pesar de que esta formaba parte del currículo (materias Metodología en la Investigación Científica e Informática Médica). Otro estudio cubano realizado por Neyra Fernández y col., ${ }^{6}$ mostró una estrategia para la incorporación de la actividad científica dentro de la carrera de Medicina, en la que se establecían actividades con complejidad creciente en todos los años de la carrera.

Nuestro estudio presenta una serie de limitaciones relacionadas con el número de estudiantes analizados y el tiempo disponible para que los estudiantes realizaran el ejercicio (contenido teórico, trabajo de campo y análisis de resultados), y el nivel de satisfacción expresado puede estar sesgado por el efecto techo y no ser representativo de la percepción de los alumnos. De todos modos, consideramos que se trata de la descripción de una experiencia educativa valiosa de un tema tan importante y complejo como la incorporación de la investigación en estudiantes de grado.

\section{CONCLUSIÓN}

La experiencia docente en la enseñanza de investigación en estudiantes de la materia Pediatría de la carrera de Medicina fue satisfactoria.

Los estudiantes reconocen la importancia de aprender a investigar durante la carrera, pero han recibido poca formación y tampoco han participado en estudios de investigación.

\section{REFERENCIAS}

1. Argimon Pallás J, Jiménez Villa J. Métodos de Investigación Clínica Y Epidemiología. 3. ${ }^{\text {ra }}$ ed. Madrid: Elsevier; 2004.

2. Arteaga Estrada M, Cruz Saldivar M. Reseña de: Las universidades modernas: espacios de investigación y docencia, de Burton R Clark. Perfiles Educativos 1999;21(8384):147-9.

3. Clark B. The Modern Integration of Research Activities with Teaching and Learning. J High Educ 1997;68(3):241-55.

4. Healey M. Linking research and teaching: exploring disciplinary spaces and the role of inquiry-based learning. In Barnett, R, ed. Reshaping the University: New Relationships between Research, Scholarship and Teaching. Berkshire: McGraw Hill/Open University Press; 2005:67-78.

5. Pizarro R, Eymann A, Rubinstein F, Belziti C, et al. Validación de un instrumento para la evaluación de la interpretación de los resultados de estudios deinvestigación en los residentes de un hospital universitario. Educ Med 2011;14(3):171-9.

6. Neyra Fernández M, BerraSocarrás M, Rodríguez Mendoza A, Rodríguez Lastra R, et al. La estrategia investigativa 
curricular en la carrera de medicina. Educ Med Super 1997;11(2):91-100.

7. Gutiérrez C, Mayta P. Publicación desde el Pre Grado en Latinoamérica: importancia, limitaciones y alternativas de solución. Cimel 2003;8(1):54-60.

8. Cabrera-Enríquez JA, Cruzado-Mendoza CC, PurizacaRosillo N, López-Samanamú RO, et al. Factores asociados con el nivel de conocimientos y la actitud hacia la investigación en estudiantes de medicina en Perú, 2011. Rev Panam Salud Pública 2013;33(3):166-73.

9. Cursiefen C, Altunbas A. Contribution of medical student research to the Medline-indexed publications of a German medical faculty. Med Educ 1998;32(4):439-40.

10. Ricoy JR, Carrasco M, Clavería LE. Educación médica e investigación. Med Clin (Internet)1999;112(7):259-63.

11. Díaz Vélez C, Manrique González LM, Galán Rodas E, Apolaya Segura M. Conocimientos, actitudes y prácticas en investigación de los estudiantes de pregrado de facultades de medicina del Perú. Acta Méd Peruana 2008;25(1):9-15.

12. Ángel-Isaza A, Botero-Suárez H, González DC, Ospina $\mathrm{LP}$, et al. Interés de los estudiantes de medicina por la investigación. Cimel 2010;15(1):9-13.

13. Fernández MJ, Rubio Olivares DY, González Sánchez R, Fundora Mirabal J, et al. La formación investigativa de los estudiantes de Medicina. Educ Med Super 2008;22(4):1-16.

14. Eymann A, Busaniche J, Durante E, Sadler A, et al. Análisis de la procedencia universitaria y desempeño en el examen de ingreso de los aspirantes a las residencias del Hospital Italiano de Bs As: 2003-2006. Rev Hosp Ital B Aires 2006;26(4):138-41.

15. Pamo-Reyna O. Una visión histórica de la participación femenina en la profesión médica. Rev Soc Peru Med Interna 2007;20(3):109-22.

16. NeumanM,QuestaU,KaufmannR.Concursoderesidencias médicas en la ciudad de Buenos Aires: importancia del género y universidad. Educ Med 2004;7(2):90-6.
17. McLemore E, Ramamoorthy S, Peterson CY, Bass BL. Women in Surgery: Bright, Sharp, Brave, and Temperate. Perm J 2012;16(3):54-9.

18. FACTS: Applicants, Matriculants, Enrollment, Graduates, $\mathrm{MD} / \mathrm{PhD}$, and Residency Applicants Data. Washington, DC: Association of American Medical Colleges; 1995-2012. [Acceso: 17 de noviembre de 2016]. Disponible en: https:// www.aamc.org/data/facts/.

19. McKinstry B. Are there too many female medical graduates? Yes. BMJ 2008;336(7647):748.

20. Burton KR, Wong IK. A force to contend with: the gender gap closes in Canadian medical schools. CMAJ 2004; 170(9):1385-6.

21. Gargiulo DA, Hyman NH, Hebert JC. Women in Surgery, Do we really understand the deterrents? Arch Surg 2006;141(4):405-7.

22. Pastor-Gosálbez MI, Belzunegui Eraso A, Pontón Merina P. Mujeres en sanidad: entre la igualdad y la desigualdad. Cuad Relac Lab 2012;30(2):497-518.

23. Coromoto Morillo Moreno M. Labor del tutor y asesor de trabajo de investigación. Experiencias e incentivos. Educere (En línea) 2009;13(47):919-30.

24. OPS. Guía para escribir un protocolo de investigación. [Acceso: 17 de noviembre de 2016]. Disponible en: http:/ / www.sld.cu/galerias/pdf/sitios/rehabilitacion-bal/ops_ protocolo.pdf.

25. Rodríguez del Águila MDM, Pérez Vicente S, del Castillo LS, Fernández Sierra MA. Cómo elaborar un protocolo de investigación en salud. Med Clin (Barc) 2007;129(8):299-302.

26. Facultad de Medicina. UBA. Plan de estudios de la carrera de medicina. [Acceso: 17 de noviembre de 2016]. Disponible en: http://www.fmed.uba.ar/grado/medicina/m_plan. htm.

27. Facultad de Medicina. UBA. Información general de la carrera de medicina. [Acceso: 17 de noviembre de 2016]. Disponible en: http://www.fmed.uba.ar/grado/ medicina/m_medicina.htm. 


\section{Anexo}

Encuesta de evaluación de la actividad de investigación

Edad: $\quad$ años. $\quad$ Sexo:

Número de materias aprobadas:

¿Ha recibido formación en metodología de la investigación durante la carrera de Medicina?

$$
\text { SÍ NO }
$$

¿Había participado previamente en un estudio de investigación durante la carrera de Medicina?

$$
\text { Sí NO }
$$

Evalúe qué importancia le adjudica a aprender herramientas para investigar durante la carrera de Medicina con un puntaje de 1 a 10 (1: mínima importancia; 10: máxima importancia).

Evalúe su grado de satisfacción de la actividad de investigación realizada durante la cursada de Pediatría con un puntaje de 1 a 10 (1: mínima satisfacción; 10: máxima satisfacción).

Mencione 3 aspectos que considere positivos de la actividad de investigación.

Mencione 3 aspectos que considere negativos de la actividad de investigación. 